Display Settings: Abstract

FASEB J. 2009 Oct;23(10):3494-505. Epub 2009 Jul 7.

\title{
Characterization of human adult stem-cell populations isolated from visceral and subcutaneous adipose tissue.
}

Baglioni S, Francalanci M, Squecco R, Lombardi A, Cantini G, Angeli R, Gelmini S,

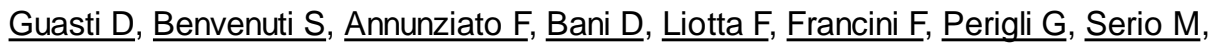
Luconi M.

Department of Clinical Physiopathology, University of Florence, Viale Pieraccini 6, 50139 Florence, Italy.

\begin{abstract}
Adipose tissue is a dynamic endocrine organ with a central role in metabolism regulation. Functional differences in adipose tissue seem associated with the regional distribution of fat depots, in particular in subcutaneous and visceral omental pads. Here, we report for the first time the isolation of human adipose-derived adult stem cells from visceral omental and subcutaneous fat (V-ASCs and S-ASCs, respectively) from the same subject. Immunophenotyping shows that plastic culturing selects homogeneous cell populations of V-ASCs and S-ASCs from the corresponding stromal vascular fractions (SVFs), sharing typical markers of mesenchymal stem cells. Electron microscopy and electrophysiological and real-time RT-PCR analyses confirm the mesenchymal stem nature of both V-ASCs and S-ASCs, while no significant differences in a limited pattern of cytokine/chemokine expression can be detected. Similar to S-ASCs, V-ASCs can differentiate in vitro toward adipogenic, osteogenic, chondrogenic, muscular, and neuronal lineages, as demonstrated by histochemical, immunofluorescence, real-time RT-PCR, and electrophysiological analyses, suggesting the multipotency of such adult stem cells. Our data demonstrate that both visceral and subcutaneous adipose tissues are a source of pluripotent stem cells with multigermline potential. However, the visceral rather than the subcutaneous ASC could represent a more appropriate in vitro cell model for investigating the molecular mechanisms implicated in the pathophysiology of metabolic disorders such as obesity.
\end{abstract}

PMID: 19584303 [PubMed - indexed for MEDLINE] Free full text

Publication Types, MeSH Terms

LinkOut - more resources 